\title{
Solar Composition of Icy Planetesimals: A New Source For Comet Nuclei?
}

\author{
Tobias Owen \\ Institute for Astronomy, University of Hawaii, 2680 Woodlawn Drive, \\ Honolulu, Hawaii 96822 USA
}

The enrichment of heavy elements on Jupiter appears to require the existence of a new class of icy planetesimal that exhibits solar relative abundances.

Prior to the Galileo probe mission, observations of methane in Jupiter's atmosphere had revealed that $\mathrm{C} / \mathrm{H}$ was approximately three times the solar ratio. This enrichment was thought to be the result of the delivery of heavy elements by icy planetesimals, which were assumed to be essentially identical to comets. However, comets are notoriously deficient in nitrogen (e.g., Geiss 1987; Krankowsky 1991) and recent upper limits on argon in three comets (Weaver et al. 2002) indicate that this element is also sub-solar relative to $O$. Hence it was assumed that Jupiter would exhibit the same deficiency in argon and nitrogen relative to carbon (Pollack and Bodenheimer 1989; Owen \& Bar-Nun 1995). Yet the mass spectrometer on the Galileo Probe clearly showed that $\mathrm{Ar}, \mathrm{Kr}, \mathrm{Xe}, \mathrm{N}$, $\mathrm{C}$, and $\mathrm{S}$ are all enriched in Jupiter's atmosphere by the same factor of $3 \pm 1$ (Niemann et al. 1998; Owen et al. 1999).

The only measured elements that were not found to be enriched were helium, which precipitates out in the deep interior, and neon, which dissolves in the helium droplets (Roulston \& Stevenson 1997). The oxygen abundance could not be measured because the probe did not go deep enough to reach levels of Jupiter's atmosphere where water - the main oxygen reservoir-was well mixed.

These results define the need for a new class of icy planetesimal, one that exhibits the solar composition of all elements less volatile than hydrogen, helium and neon. If these solar composition icy planetesimals (SCIPs) built the cores of all of the giant planets, they must have been the most abundant form of solid matter in the early solar system (Owen \& Encrenaz 2003). How did they form?

The deficiency of nitrogen in comets is ascribed to the fact that this element is expected to be present primarily in the form of $\mathrm{N}_{2}$, in both the interstellar medium from which the solar system originated and in the outer solar nebula, the home, if not the birthplace, of the comets. $\mathrm{N}_{2}$ is highly volatile and does not readily condense on itself or get trapped by forming ice, as graphically demonstrated by the presence of nitrogen compounds in dark, interstellar clouds in which CO is highly depleted (e.g., Caselli et al. 2003). Laboratory experiments on the trapping of argon, $\mathrm{N}_{2}$, and $\mathrm{CO}$ in forming amorphous ice also reveal this difference in behavior (Owen \& Bar-Nun 1995).

Thus in order to deliver nitrogen and argon to Jupiter, the icy planetesimals that carried them must have formed at low temperatures-below $27 \mathrm{~K}$ if the condensing water vapor formed amorphous ice, and below $38 \mathrm{~K}$ if it made clathrate hydrates. Evidently, low temperature is the key to making these SCIPs. 
There seem to be two possibilities. Either they were made as amorphous ice in the ISM and then transported into the solar nebula without subliming, or they were made as clathrate hydrates from crystalline ice that condensed within the nebula. In the latter case, they will exhibit $\geq 9$ times solar oxygen, in order to have enough water molecules to build the crystal cages that trap the molecules. Jupiter would then exhibit at least 9 times the solar value of $\mathrm{O} / \mathrm{H}$, instead of the 3 times enrichment predicted for the amorphous case (Gautier et al. 2001a, b).

Could there be fragments of SCIPs in our population of comets? To find them we need to look for solar values of $\mathrm{Ar} / \mathrm{O}$ and $\mathrm{N} / \mathrm{O}$, and interstellar values of $\mathrm{N}_{2} / \mathrm{O}$, which we could only expect in dynamically new comets coming in from the Oort cloud for the first time. The easiest observation is a search for the predicted high abundance of $\mathrm{N}_{2}$, in the form of $\mathrm{N}_{2}^{+}$in the ion tails of these comets. It is important to note, however, that the ratio $\mathrm{N}_{2}^{+} / \mathrm{CO}^{+}$, which is commonly measured, contains the ambiguity of the $\mathrm{CO}$ abundance, which is known to vary from comet to comet (Crovisier \& Bockelée-Morvan 1999). Thus it is critical to obtain $\mathrm{CO} / \mathrm{H}_{2} \mathrm{O}$ in the comet and to derive $\mathrm{N}_{2} / \mathrm{H}_{2} \mathrm{O}$ for comparison with expected interstellar values. Although $\mathrm{N}_{2}$ is not observed directly in the ISM, up to $90 \%$ of the total nitrogen may be in this form, based on studies of $\mathrm{N}_{2} \mathrm{H}^{+}$ and $\mathrm{NH}_{3}$ (van Dishoeck et al. 1993).

\section{References}

Caselli, P., et al. 2003, presented at this conference.

Crovisier, D., \& Bockelée-Morvan, D. 1999, Space Sci.Rev., 90, 19

Gautier, D., Hersant, F., Mousis, O., \& Lunine, J. I. 2001a, ApJ, 550, L227

Gautier, D., Hersant, F., Mousis, O., \& Lunine, J. I. 2001b, ApJ, 559, L183

Geiss, J. 1987, A\&A, 187, 859

Krankowsky, D., 1991, in Comets in the Post-Halley Era, eds. R. L. Newburn, M. Neugebauer, \& J. Rahe (Dordrecht: Kluwer), 855

Niemann, H. B., et al. 1998, J. Geophys. Res., 103, 22831

Owen, T., \& Bar-Nun, A. 1995, Icarus, 116, 215

Owen, T., \& Encrenaz, Th. 2003, Space Sci.Rev., 106, 121

Owen, T., Mahaffy, P., Niemann, H. B., Atreya, S., Donahue, T., Bar-Nun, A., \& de Pater, I. 1999, Nature, 402, 269

Pollack, J. B., \& Bodenheimer, P. 1989, in Origin and Evolution of Planetary and Satellite Atmospheres, eds. S. K. Atreya, J. B. Pollack, \& M. S. Matthews (Tucson: Univ. Arizona Press), 564

Roulston, M. J., \& Stevenson, D. J. 1997, EOS Trans., AGU 76 (46) Fall Meeting Suppl. $\mathrm{F}, 343$

van Dishoek, E. F., Blake, G. A., Draine, B. T., \& Lunine, J. I. 1993, in Protostars and Planets III, eds. E. H. Levy \& J. I. Lunine (Tucson: Univ. Arizona Press), 163

Weaver, H. A., et al. 2002, ApJ, 576, L95 